\title{
A compelling story of disruptive physician behaviour
}

\author{
Surgical Heights \\ Paul E. Hardy MD \\ First Choice Books; 2009. \\ www.surgicalheights.com
}

I $\mathrm{t}$ is a great pleasure to read wellcrafted fiction written by physicians about physicians. The accuracy of the culture, the insights into the nuances of character and plot, and the normalization of what others find bizarre all combine to create realistic and genuine novels.

Dr. Paul Hardy, a general surgeon practising in western Canada, can count himself amongst the members of the cadre of talented physician-writers. His novel, Surgical Heights, which shares the complex and painful story of Dr. Jim Smythe, is focused on the theme of disruptive physician behaviour.

This theme is of increasing relevance and interest across Canada and is also frequently misunderstood. Physicians do not display disruptive behaviour at rates higher than other

\section{Stories are an important tool for sharing}

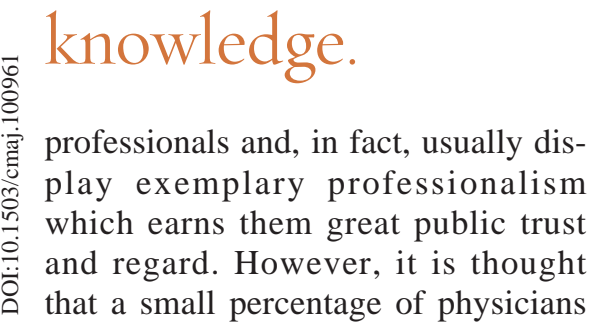

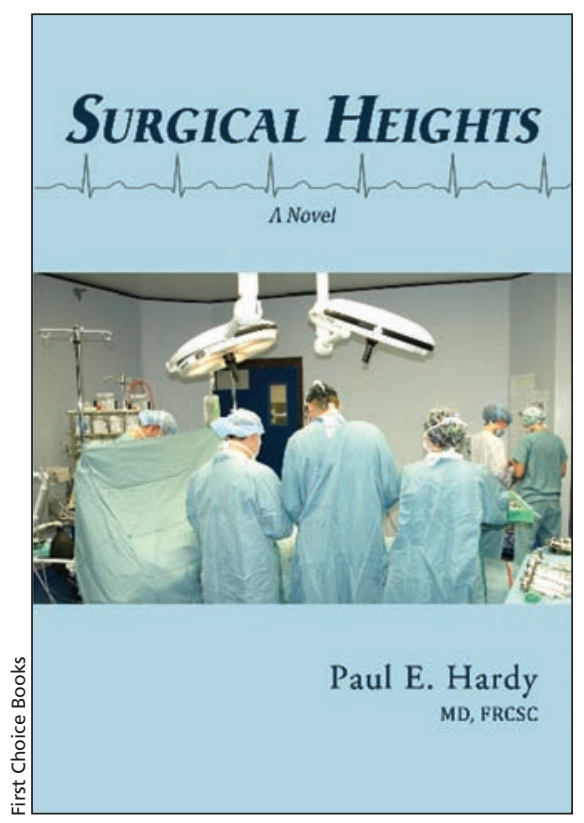

display behaviour that can, ultimately, interfere with the delivery of quality health care and that the vast majority of these physicians are living with an undiagnosed and treatable form of mental illness or substance use. ing end of patient and peer complaints, runs into difficulty with his chief of staff and medical licensing authority, and eventually requires a dramatic and serious intervention in order to protect patients and, sadly, himself.

Hardy does an excellent job of telling such a story and anchors the outcome in frank realism. Smythe requires an inpatient psychiatric admission and the loss of his privileges to begin the process of developing insight and moving toward recovery. $\mathrm{He}$ is enrolled in a physician health program with mandatory monitoring and begins to make changes to his lifestyle that better promote health rather than extreme altruism.

Physician health programs are increasingly involved in the care and support of colleagues with disruptive behaviour, mental illness or physical health problems. The rates are not increasing; rather, the profession's willingness to challenge dogma, move beyond stigma and promote collegiality (even in the most difficult of times) is. Stories such as Surgical Heights are powerful and important tools of sharing knowledge about physician health and are an important component of any curriculum in the area.

Smythe is such a physician. He has dedicated his career to the service of others and is a well-regarded general surgeon. However, in a few short months, he begins to be on the receiv-

\section{Derek Puddester MD}

Director

Faculty Wellness Program

University of Ottawa

Ottawa, Ont. 